\section{Uso da metodologia de relacionamento de bases de dados para qualificação da informação sobre mortalidade infantil nos municípios de Pernambuco}

\section{The use of the interrelation of data bases to improve information on child mortality in municipalities in the Brazilian State of Pernambuco}

Antônio da Cruz Gouveia Mendes 1

Mirella Muzzi de Lima 2

Domício Aurélio de Sá 3

Luiz Cláudio de Souza Oliveira 4

Lívia Teixeira de Souza Maia 5

1-5 Centro de Pesquisas Aggeu Magalhães. Fundação Oswaldo Cruz (CPqAM - Fiocruz). Av. Prof. Moraes Rego, s.n. CP 7472. Campus UFPE. Recife, PE, Brasil. CEP: 50.670-420.

E-mail: amendes@cpqam.fiocruz.br

\begin{abstract}
Objectives: to ascertain the degree of completeness and consistency of data in the Mortality Information System (SIM) and the Live Births Information System (Sinasc) in Pernambuco and to analyze the quality of information on child mortality obtained using these systems.

Methods: the SIM and Sinasc databases were combined using the linkage technique and deterministic and probabilistic methods. The data base obtained was analyzed according to the type of relation, the child mortality component and the demographic scope of the municipality of residence. Analysis of the fields filled in in the registers considered variables common to both systems.

Results: deterministic linkage helped greatly to identify true pairs of neonatal deaths, while the probabilistic method allowed a large portion of the postneonatal records to be recovered, especially in smaller municipalities. It was also found that there was a significant improvement in the completeness of the information analyzed, with the Sinasc contributing more to improve the information contained in the SIM.

Conclusion: linkage made it possible to reduce the percentage of empty fields for all variables under study to less than 1\% in both systems, showing that this is an important tool for ascertaining and improving the quality of information on child mortality.
\end{abstract}

Key words Infant mortality, Information systems, Vital statistics

\section{Resumo}

Objetivos: identificar a completitude e a consistência dos dados do Sistema de Informação sobre Mortalidade (SIM) e do Sistema de Informação sobre Nascidos Vivos (Sinasc) em Pernambuco e analisar a qualidade das informações sobre mortalidade infantil obtida a partir desses sistemas.

Métodos: relacionou-se as bases de dados do SIM e do Sinasc por meio da técnica de linkage, utilizando os métodos determinístico e probabilístico. O banco de dados obtido foi analisado segundo o tipo de relacionamento, componente da mortalidade infantil e porte populacional dos municípios de residencia. Para a análise do preenchimento dos campos dos registros foram consideradas as variáveis comuns aos dois sistemas.

Resultados: o linkage deterministico contribuiu fortemente para a identificação dos pares verdadeiros dos óbitos neonatais, enquanto que o método probabilístico permitiu o resgate de grande parte dos registros pós-neonatais, especialmente em municípios menores. Além disso, foi observado um aumento significativo na completitude das informações analisadas, com uma maior contribuição do Sinasc para o incremento de informações no SIM.

Conclusão: o linkage possibilitou uma redução no percentual de campos ignorados de todas as variáveis analisadas para menos de 1\%, em ambos os sistemas, mostrando-se uma importante ferramenta para o conhecimento e aprimoramento da qualidade das informações sobre mortalidade infantil.

Palavras-chave Mortalidade infantil, Sistemas de informação, Estatísticas vitais 


\section{Introdução}

Apesar do uso consagrado do coeficiente de mortalidade infantil como excelente indicador da saúde pública, a maioria dos países em desenvolvimento não dispõe de informações com qualidade satisfatória para calcular este evento. Com isso, torna-se difícil a obtenção de dados reais em virtude das limitações presentes nas fontes de informações, ${ }^{1}$ o que compromete a análise dos fatores que influenciam essa mortalidade e, consequentemente, dificulta o planejamento das ações de intervenção. ${ }^{2}$

Para minimizar esse problema, muitos estudos foram e ainda são realizados com o intuito de analisar não apenas as fontes primárias de informação como também os próprios sistemas informatizados em diversas localidades, empregando diferentes metodologias.3-7

Os resultados dos diversos estudos realizados sobre a qualidade da informação em saúde em diferentes regiões do país mostram que mesmo com o progressivo aperfeiçoamento do Sistema de Informação sobre Mortalidade (SIM) e do Sistema de Informação sobre Nascidos Vivos (Sinasc), com o crescente nível de cobertura e a melhoria da qualidade dos dados, 1,8 ainda não é possível calcular o coeficiente de mortalidade infantil pelo método direto, principalmente nas Regiões Norte e Nordeste, 8 bem como em vários municípios brasileiros, com menor número de habitantes. ${ }^{9}$ Os municípios de maior porte do eixo Sul-Sudeste apresentam melhores informações quando comparados aos menores. ${ }^{9}$ Esses achados estão de acordo com o estudo de Luiz et al.,10 que estudaram os diferenciais intermunicipais de condições de vida e saúde, identificando diferentes níveis de desenvolvimento de acordo com o porte populacional.

Os fatores que comprometem a qualidade da informação de ambos os sistemas é a subinformação, ${ }^{11}$ a subnotificação, ${ }^{11}$ o sub-registro ${ }^{11,12}$ e a baixa confiabilidade dos dados $4,13,14$ pela presença de variáveis ignoradas ou não preenchidas 8 e ainda no SIM, erro na classificação do tipo de óbito e da causa básica da morte.

Um dos fatores que comprometem a qualidade da informação disponibilizada pelos sistemas é a falha no preenchimento da Declaração de Óbito (DO), 6,7 incluindo o erro na classificação da causa básica da morte, a subnotificação, 11 o subregistro 11,12 e presença de variáveis ignoradas ou não preenchidas. ${ }^{8}$

Além da subinformação e da baixa confiabilidade dos dados, $4,13,14$ o preenchimento dos campos das declarações de nascimentos (DN) e óbitos (DO), que representa a completitude dos registros, um dos atributos de qualidade, sendo esta a segunda dimensão de qualidade mais avaliada, apresentando uma diversidade de resultados não apenas entre os sistemas analisados, como também entre as variáveis dentro de um mesmo sistema e entre diferentes localidades. ${ }^{1,14-16}$

Uma opção de relativo baixo custo operacional ${ }^{14,17}$ para a análise da qualidade do preenchimento de dados é a comparação entre sistemas de informação, a partir da aplicação da metodologia de relacionamento de bases de dados ou linkage, com excelente viabilidade, $17-19$ trazendo como vantagem a possibilidade de análises mais detalhadas da qualidade de dados oficiais ${ }^{8}$ e ganho de informações comuns aos sistemas relacionados. ${ }^{20,21}$ Além disso, permite a formulação de críticas à qualidade dos sistemas, servindo como estímulo para o aperfeiçoamento dos mesmos. ${ }^{21}$

No estudo da mortalidade infantil esta metodologia tem sido empregada e se mostrado uma ferramenta útil. Nestes casos é comum o compartilhamento das informações dos dois sistemas específicos de registros de nascimentos e óbitos, Sinasc e SIM, respectivamente. 22

Neste sentido, esse trabalho teve como objetivo identificar a completitude e a consistência entre os dados do SIM e do Sinasc em Pernambuco e analisar a qualidade das informações sobre mortalidade infantil obtida a partir desses sistemas, segundo porte dos municípios.

\section{Métodos}

Estudo descritivo de corte transversal no qual foi realizado o relacionamento das bases de dados dos dois sistemas para avaliação da dimensão completitude, o SIM, com 3267 registros de óbitos em menores de um ano, notificados em 2005, e do Sinasc com registro de 301.834 nascidos vivos nos anos 2004 e 2005 no Estado de Pernambuco.

Para o linkage das bases foram aplicados os métodos de relacionamento determinístico e probabilístico. No primeiro, foi utilizada como variávelchave o número da declaração de nascido vivo e no segundo, as variáveis foram: nome da mãe, município de residência, data de nascimento e sexo da criança. Para execução do relacionamento probabilístico das bases foi utilizado o programa ReclinkIII, ${ }^{21}$ que permitiu a aplicação do algoritmo soundex para a codificação do primeiro e último nomes das mães, a fim de minimizar o efeito de erros ou diferenças de grafia nos dois sistemas sobre o pareamento. Foram considerados pares apenas os registros que apresentaram todas as variáveis coincidentes, sendo ainda realizada uma revisão de todos 
os pares identificados.

Foram também relacionados os óbitos fetais com o Sinasc, a fim de identificar possíveis erros de classificação.

A base de dados resultante foi analisada segundo o tipo de relacionamento, componentes, neonatal e pós-neonatal, e, porte populacional dos municípios de residência.

$\mathrm{Na}$ estratificação do Estado por porte municipal, buscou-se classificar os municípios nas categorias pequeno, médio e grande porte, partindo do pressuposto da existência de diferentes níveis de qualidade dos sistemas de informação. 9,10 Para a construção do porte populacional os municípios foram agregados em estratos de acordo com o número de residentes: porte 1 (municípios com menos de 20.000 habitantes); porte 2 (20.000 a 49.999); porte $3(50.000$ a $99.999)$; porte 4 (100.000 a 499.999$)$; porte 5 (500.000 a 1 milhão); e o porte 6 , com mais de 1 milhão de habitantes, no qual encontra-se a capital pernambucana.

$\mathrm{Na}$ análise da completitude foram considerados apenas os campos dos registros pareados: sexo, peso ao nascer, raça/cor, idade da mãe, instrução da mãe tipo de gravidez, tipo de parto e duração da gestação. Para esta análise foram consideradas informações incompletas aquelas cujos campos não estavam preenchidos e aquelas preenchidas com o código de ignorado. Além da completitude, foram calculadas as proporções de campos sem informação em relação ao total de existentes.

Este estudo é parte integrante da pesquisa intitulada "Integração de Banco de Dados como Metodologia para Melhoria do Acesso e Qualidade da Informação da Gestão em Epidemiologia" financiada pelo Ministério da Saúde mediante convênio com a Secretaria de Vigilância à Saúde e aprovada pelo Comitê de Ética em Pesquisa da Fiocruz /PE sob o registro CEP/CPqAM/Fiocruz: 67/07, CAAE: 0067.0.095.000-07.

\section{Resultados}

Os municípios considerados de pequeno porte, com menos de 50.000 habitantes (portes 1 e 2), correspondem a $82,7 \%$ do Estado; os de médio porte (3 e 4) representam $16,2 \%$; e os de grande porte populacional (>500 mil habitantes), são representados por apenas dois municípios (portes 5 e 6 ).

O linkage realizado pelo método probabilístico entre os óbitos fetais e nascidos vivos identificou 31 erros de classificação. Estes óbitos mal classificados foram acrescentados aos óbitos não fetais no SIM, totalizando 3298 óbitos em 2005. Destes, 1934 (59\%) foram pareados com os nascidos vivos do
Sinasc (2004 e 2005) mediante relacionamento determinístico; 949 (29\%) por meio de pareamento probabilístico e 415 (13\%) óbitos não foram localizados no Sinasc.

A Figura 1 apresenta a distribuição percentual dos registros do SIM e do Sinasc pareados e não pareados segundo tipo de linkage e porte municipal, mostrando que quanto maior o porte municipal, maior identificação dos pares, $90 \%$ no município de porte 6.

Quanto ao linkage probabilístico, observou-se um incremento de pares superior a $30 \%$ nos portes 1 a 4. Os registros não pareados apresentam uma variação de $14,8 \%$ no porte 4 a 2,6\% no município de porte 6 .

$\mathrm{Na}$ análise do tipo de relacionamento por componente da mortalidade infantil observou-se que o linkage determinístico conseguiu maior pareamento (quase $70 \%$ dos óbitos neonatais). Com a metodologia probabilística, a maior proporção de pareamento foi no componente pós-neonatal $(44,5 \%)$. Quanto aos óbitos infantis não pareados, $11 \%$ neonatais e $15,6 \%$ pós-neonatais, não foram encontrados registros na base de dados do Sinasc.

$\mathrm{Na}$ análise por porte populacional dos municípios, o componente neonatal obteve menor percentual de pares formados pelo método determinístico (nos portes 1 a 4), com variação de 63,9\% no porte 1 a $95,6 \%$ no porte 6 . No método probabilístico a situação foi inversa, variando de $25 \%$ no porte 4 a $2,9 \%$ no porte 6 .

Os registros pós-neonatais apresentaram percentuais mais elevados em relação aos pares formados probabilisticamente, quando analisados os municípios de menor porte ( 1 a 4), e o de maior porte (6) destacou-se com $89,5 \%$ dos pares obtidos pelo linkage determinístico (Tabela 1).

De acordo com a Tabela 2, antes do relacionamento dos bancos, o SIM apresentava $89,9 \%$ de completitude geral. Com exceção da variável sexo, todas as demais apresentaram menos de $90 \%$ de completitude, sendo a instrução da mãe a variável com pior nível de preenchimento. Já o Sinasc apresentou um grau de completitude de $99,2 \%$, com exceção das variáveis: instrução da mãe $(98,4 \%)$ e raça/cor $(97,1 \%)$.

Com o método de relacionamento dos bancos dos dois sistemas foi possível resgatar um considerável número de campos não preenchidos, de maneira mais significativa do Sinasc para o SIM, embora este último tenha incrementado ao Sinasc 64 registros referentes à raça/cor e 29 para instrução da mãe. Após o linkage foi possível alcançar um grau de completitude de $99,7 \%$, em ambos os sistemas. 
Distribuição percentual dos registros do Sistema de Informação sobre Mortalidade e do Sistema de Informação sobre Nascidos Vivos pareados e não pareados segundo tipo de linkage e porte municipal.

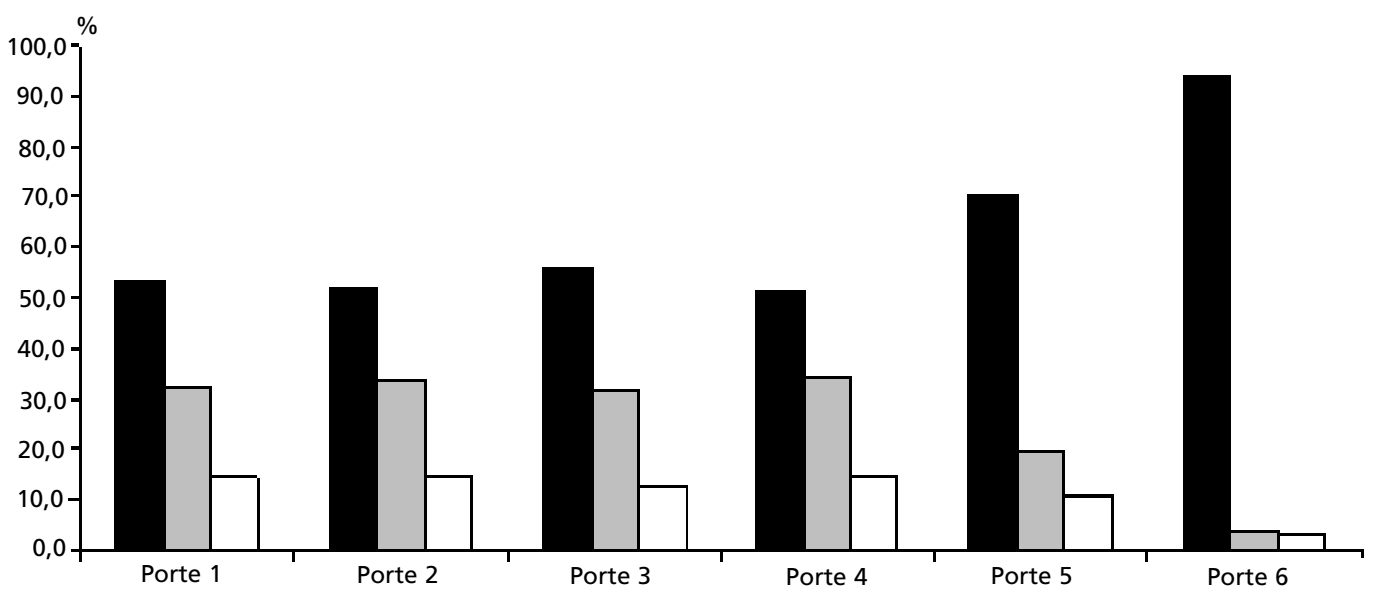

Determinístico $\square$ Probabilístico $\square$ Não Pareado

Fonte: Sistema de Informação sobre Mortalidade; Sistema de Informação sobre Nascidos Vivos.

Tabela 1

Distribuição dos números absolutos de óbitos em menores de um ano, segundo porte municipal, tipo de linkage e componente da mortalidade infantil, Pernambuco, 2005.

\begin{tabular}{|c|c|c|c|c|c|c|c|c|c|c|c|c|c|c|}
\hline \multirow{3}{*}{$\begin{array}{l}\text { Componente / tipo de } \\
\text { linkage }\end{array}$} & \multicolumn{14}{|c|}{ Porte populacional } \\
\hline & \multicolumn{2}{|c|}{ Porte 1} & \multicolumn{2}{|c|}{ Porte 2} & \multicolumn{2}{|c|}{ Porte 3} & \multicolumn{2}{|c|}{ Porte 4} & \multicolumn{2}{|c|}{ Porte 5} & \multicolumn{2}{|c|}{ Porte 6} & \multicolumn{2}{|c|}{ Total } \\
\hline & $\mathrm{n}$ & $\%$ & $\mathrm{n}$ & $\%$ & $\mathrm{n}$ & $\%$ & $\mathrm{n}$ & $\%$ & $\mathrm{n}$ & $\%$ & $\mathrm{n}$ & $\%$ & $\mathrm{n}$ & $\%$ \\
\hline Óbitos em menores de um ano & 622 & 100,0 & 934 & 100,0 & 600 & 100,0 & 587 & 100,0 & 166 & 100,0 & 388 & 100,0 & 3.298 & 100,0 \\
\hline Determinístico & 332 & 53,4 & 494 & 52,9 & 335 & 55,8 & 301 & 51,3 & 117 & 70,5 & 364 & 93,8 & 1.943 & 58,9 \\
\hline Probabilístico & 202 & 32,5 & 313 & 33,5 & 189 & 31,5 & 199 & 33,9 & 32 & 19,3 & 14 & 3,6 & 949 & 28,8 \\
\hline Não Pareado & 88 & 14,1 & 136 & 14,6 & 76 & 12,7 & 87 & 14,8 & 17 & 10,2 & 10 & 2,6 & 414 & 12,6 \\
\hline Óbitos neonatais* & 379 & 100,0 & 548 & 100,0 & 391 & 100,0 & 400 & 100,0 & 104 & 100,0 & 274 & 100,0 & 2096 & 100,0 \\
\hline Determinístico & 242 & 63,9 & 365 & 66,6 & 261 & 66,8 & 248 & 62,0 & 86 & 82,7 & 262 & 95,6 & 1464 & 69,8 \\
\hline Probabilístico & 86 & 22,7 & 121 & 22,1 & 87 & 22,3 & 100 & 25,0 & 12 & 11,5 & 8 & 2,9 & 414 & 19,8 \\
\hline Não Pareado & 51 & 13,5 & 71 & 13,0 & 43 & 11,0 & 52 & 13,0 & 6 & 5,8 & 4 & 1,5 & 227 & 10,8 \\
\hline Óbitos pós-neonatais & 243 & 100,0 & 386 & 100,0 & 209 & 100,0 & 187 & 100,0 & 62 & 100,0 & 114 & 100,0 & $1202^{* *}$ & 100,0 \\
\hline Determinístico & 90 & 37,0 & 129 & 33,4 & 74 & 35,4 & 53 & 28,3 & 31 & 50,0 & 102 & 89,5 & 479 & 39,9 \\
\hline Probabilístico & 116 & 47,7 & 192 & 49,7 & 102 & 48,8 & 99 & 52,9 & 20 & 32,3 & 6 & 5,3 & 535 & 44,5 \\
\hline Não Pareado & 37 & 15,2 & 65 & 16,8 & 33 & 15,8 & 35 & 18,7 & 11 & 17,7 & 6 & 5,3 & 187 & 15,6 \\
\hline
\end{tabular}

Fonte: Sistema de Informação sobre Mortalidade (SIM); * Os 31 óbitos registrados no SIM como fetal e pareados com o Sinasc; * Deste total, um óbito apresentou município ignorado e não foi classificado por portes. 
Distribuição das frequências ignoradas das variáveis comuns ao Sistema de Informação sobre Mortalidade e ao Sistema de Informação sobre Nascidos Vivos, antes e após o linkage dos bancos de dados, Pernambuco, 2004/2005.

\begin{tabular}{|c|c|c|c|c|c|c|}
\hline \multirow{3}{*}{ Variáveis } & \multicolumn{4}{|c|}{ Pré-linkage } & \multicolumn{2}{|c|}{ Pós-linkage } \\
\hline & \multicolumn{2}{|c|}{ SIM } & \multicolumn{2}{|c|}{ Sinasc } & \multicolumn{2}{|c|}{ SIM / Sinasc } \\
\hline & $\mathrm{n}$ & $\%$ & $\mathrm{n}$ & $\%$ & $\mathrm{n}$ & $\%$ \\
\hline Sexo & 15 & 0,5 & 13 & 0,5 & 10 & 0,3 \\
\hline Peso ao nascer & 391 & 13,6 & 20 & 0,7 & 14 & 0,5 \\
\hline Raça/Cor & 379 & 13,1 & 85 & 2,9 & 21 & 0,7 \\
\hline Idade da mãe & 336 & 11,7 & 2 & 0,1 & 1 & 0,0 \\
\hline Instrução da mãe & 476 & 16,5 & 47 & 1,6 & 18 & 0,6 \\
\hline Tipo de gravidez & 307 & 10,6 & 0 & 0,0 & 0 & 0,0 \\
\hline Tipo de parto & 309 & 10,7 & 2 & 0,1 & 1 & 0,0 \\
\hline Duração da gestação & 345 & 12,0 & 18 & 0,6 & 8 & 0,3 \\
\hline Total de registros incompletos & 2558 & 11,1 & 187 & 0,8 & 73 & 0,3 \\
\hline
\end{tabular}

Fonte: $\mathrm{SIM}=$ Sistema de Informação sobre Mortalidade; Sinasc= Sistema de Informação sobre Nascidos Vivos.

\section{Discussão}

A aplicação do método de linkage nos bancos do SIM e do Sinasc visa identificar e resgatar informações que estão presentes em um sistema e ausentes ou incompletas no outro.

A distribuição dos óbitos infantis segundo os componentes neonatal e pós-neonatal e o porte municipal possibilitou o conhecimento das desigualdades existentes entres os municípios de Pernambuco, em relação à qualidade da informação produzida.

Com relação à qualidade da informação, esperase uma melhor qualidade dos registros vitais de nascimentos e óbitos neonatais, considerando que a maioria desses eventos acontece em hospitais.

Neste estudo, embora os óbitos neonatais, quando comparados aos pós-neonatais, tenham apresentado um maior número de registros pareados deterministicamente, indicando uma melhor qualidade das informações, os valores encontram-se ainda aquém do esperado. Apenas os municípios com população acima de $500 \mathrm{mil}$ habitantes obtiveram mais de $80 \%$ dos óbitos neonatais pareados através do linkage determinístico.

Os resultados apresentados no atual estudo para os municípios de pequeno e médio portes se comportaram de maneira muito semelhante aos encontrados por Frias et al., ${ }^{9}$ que verificaram maiores problemas na qualidade das informações em municípios menores. Mostraram também que quanto maior o município, maiores são os registros pareados deterministicamente e menor a ocorrência de não pares, e inversamente, quanto menor o município, maior é a contribuição do linkage probabilístico e um número maior de registros não são pareados.

Diante do exposto, evidencia-se a necessidade da adoção de estratégias prioritárias, principalmente para os municípios com população inferior a $500 \mathrm{mil}$ habitantes, os quais, em Pernambuco, concentraram $83,2 \%$ dos óbitos de menores de 1 ano, refletindo diretamente no coeficiente de mortalidade infantil do Estado.

Dentre as estratégias já adotadas visando à melhoria da qualidade das informações, a técnica de linkage deveria ser uma das medidas a ser adotada na rotina dos serviços municipais de saúde, e a gestão estadual poderia incentivar e utilizá-la para o relacionamento dos seus bancos de dados consolidados. Outra estratégia, importante para o aprimoramento da qualidade dos sistemas de informação, é um maior investimento na área de vigilância à saúde, garantindo infra-estrutura adequada e condições de trabalho para as equipes envolvidas nas atividades de coleta dos dados, processamento, análises e retroalimentação das informações.

Quanto à completitude, a contribuição, como era esperada, ${ }^{9}$ ocorreu de modo mais expressivo do Sinasc para o SIM, em virtude dos próprios objetivos dos sistemas.

Outros estudos, em localidades distintas, revelaram percentuais de campos incompletos nas declarações de óbitos de menores de um ano bastante superiores aos encontrados em Pernambuco. 15,23 Tal fato reforça a potencialidade da utilização do relacionamento dos bancos de dados como uma impor- 
tante estratégia para o aprimoramento da qualidade dos sistemas nacionais de informação em saúde.

Como os resultados apontaram, o linkage possibilitou o resgate de um grande número de informações que estavam incompletas em ambos os sistemas, além de identificar erros de classificação dos óbitos que modificam os indicadores de mortalidade infantil do Estado. Neste sentido, esta técnica se configura como excelente ferramenta para melhoria da qualidade das informações produzidas, além de favorecer a dinâmica dos serviços de vigilância, sobretudo referentes às estatísticas vitais.

\section{Referências}

1. Andrade CLT, Szwarcwald CL. Desigualdades socioespaciais da adequação das informações de nascimentos e óbitos do Ministério da Saúde, Brasil, 2000-2002. Cad Saúde Pública. 2007; 23: 1207-16.

2. Santa Helena ET, Rosa MB. Avaliação da qualidade das informações relativas aos óbitos em menores de um ano em Blumenau, 1998. Rev Bras Saúde Mater Infant. 2003; 3 : $75-83$.

3. Mello-Jorge MHP, Laurenti R, Gotlieb SLD. Análise da qualidade das estatísticas vitais brasileiras: a experiência de implantação do SIM e do SINASC. Ciên Saúde Coletiva. 2007; 12: 643-54.

4. Drumond EF, Machado CJ, França E. Subnotificação de nascidos vivos: procedimentos de mensuração a partir do Sistema de Informação Hospitalar. Rev Saúde Pública. 2008; 42: 55-63.

5. Haraki CAC, Gotlieb SLD, Laurenti R. Confiabilidade do Sistema de Infomações sobre Mortalidade em município do sul do Estado de São Paulo. Rev Bras Epidemiol. 2005; 8: 19-24

6. Pedrosa LDCO, Sarinho SW, Ordonha MR. Análise da qualidade da informação sobre cauda básica de óbitos neonatais registrados no Sistema de Informação sobre Mortalidade: um estudo para Maceió, Alagoas, Brasil, 2001-2002. Cad Saúde Pública. 2007; 23: 2385-95.

7. Brasil. Ministério da Saúde. Secretaria de Vigilância em Saúde. Departamento de Análise de Situação em Saúde. Saúde Brasil 2006: uma análise da desigualdade em saúde. Brasília, DF; 2006.

8. Boing AF, Boing AC. Mortalidade infantil por causas evitáveis no Brasil: um estudo ecológico no período 2000 2002. Cad Saúde Pública. 2008; 24: 447-55.

9. Frias PG, Pereira PMH, Andrade CLT, Lira PIC, Szwarcwald CL. Avaliação da adequação das informações de mortalidade e nascidos vivos no Estado de Pernambuco, Brasil. Cad Saúde Pública. 2010; 26: 671-81

10. Luiz OC, Heimann LS, Boaretto RC, Pacheco AG, Pessoto UC, Ibanhes LC, Castro IEN,Kayano J, Juqueira V, Rocha JL, Cortizo CT, Telesi Jr E. Diferenciais intermunicipais de condições de vida e saúde: construção de um indicador composto. Rev Saúde Pública. 2009; 43: 115-22.

11. Brasil. Ministério da Saúde. Sistemas de Informações sobre Mortalidade (SIM) e Nascidos Vivos (Sinasc) para os profissionais do Programa Saúde da Família. 2 ed. Brasília, DF; 2004.
12. Romero DE, Cunha CB. Avaliação da qualidade das variáveis epidemiológicas e demográficas do Sistema de Informação sobre Nascidos Vivos, 2002. Cad Saúde Pública. 2007; 23: 701-14.

13. Silva AAM, Ribeiro VS, Borba Júnior AF, Coimbra LC, Silva RA. Avaliação da qualidade dos dados dos Sistemas de Informações sobre Nascidos Vivos em 1997-1998. Rev Saúde Pública. 2001; 35: 508-14.

14. Theme Filha MM, Gama SGN, Cunha CB, Leal MC. Confiabilidade do Sistema de Informações sobre Nascidos Vivos hospitalares no município do Rio de Janeiro, 19992001. Cad Saúde Pública. 2004; 20 (Supl. 11): 83-91.

15. Soares JAS, Horta FMB, Caldeira AP. Avaliação da qualidade das informações em declarações de óbitos infantis. Rev Bras Saúde Mater Infant. 2007; 7: 289-95.

16. Camargo Júnior KR, Coeli CM. Reclink: aplicativo para o relacionamento de bases de dados, implementando o método probabilistic record linkage. Cad Saúde Pública. 2000; 16: 439-47.

17. Machado CJ. A literature review of record linkage procedures focusing on infant health outcomes. Cad Saúde Pública. 2004; 20: 362-71.

18. Santa Helena ET, Sousa CA, Silva CA. Fatores de risco para mortalidade neonatal em Blumenau, Santa Catarina: linkage entre bancos de dados. Rev Bras Saúde Mater Infant. 2005; 5: 209-17.

19. Cascão AM, Kale PL. Relacionamento das bases de dados de amputação de membros inferiores por Diabetes Mellitus: uma estratégia para melhoria da qualidade da informação sobre causas de morte no Estado do Rio de Janeiro. Cad Saúde Coletiva. 2006; 14: 361-74.

20. Nascimento EMR, Costa MCN, Mota ELA, Paim JS. Estudo de fatores de risco para óbitos de menores de um ano mediante compartilhamento de bancos de dados. Cad Saúde Pública. 2008; 24: 2593-602.

21. Camargo JR KR, Coeli CM. Reclink: aplicativo para o relacionamento de banco de dados implementando o método probabilistic record linkage. Rio de Janeiro 2007 Versão: 3.1.6.3160. Disponível em: http://paginas.terra.com.br/ educacao/kencamargo/InstalaRL3.zip

22. Morais Neto OL, Barros MBA. Fatores de risco para mortalidade neonatal e pós-neonatal na Região CentroOeste do Brasil: linkage entre bancos de dados de nascidos vivos e óbitos infantis. Cad Saúde Pública. 2000; 16: 477 85 
23. Correia LOS, Lopes CGV, Sarmento MAS. Avaliação da completitude do preenchimento dos campos essenciais do Sistema de Informação sobre Mortalidade, Alagoas, 2006. Secretaria de Saúde do Estado de Alagoas. Bol Vigil Saúde.

Recebido em 30 de abril de 201

Versão final apresentada em 7 de fevereiro de 2012

Aprovado em 25 de junho de 2012
Ano 2, $\mathrm{n}^{\mathrm{o}}$ 1, agosto, 2008. Disponível em: http://www.saude.al.gov.br/sites/default/files/avaliacao_da completitude_das_informacoes_do_sim.pdf 